\title{
Performance Analysis of an Asymmetrical Phase-Converter-Fed Induction Motor
}

\author{
Sujit Kumar Biswas, Senior Member, IEEE, Chandan Chakraborty, \\ Biswarup Basak, Member, IEEE, and Debi Prasad Sen Gupta
}

\begin{abstract}
Of the various types of single-phase-to-three-phase static converters used to interface a three-phase motor to singlephase supply, one simple type uses an open delta connection of a sinusoidal supply and a pulsewidth modulated (PWM) waveform to feed the motor. This paper presents an analysis of the performance of the three-phase motor when fed from such an asymmetrical supply. The analysis is based on the steady-state approximate-equivalent circuit of the motor, using the method of symmetrical components. The analysis is directed toward prediction of motor phase and line currents, along with the nature of generated torque. The computer-simulated waveforms are presented and compared with results from tests on a laboratory setup.
\end{abstract}

Index Terms - Harmonic analysis, induction motor drives, phase conversion, simulation.

\section{INTRODUCTION}

$\mathbf{T}$ WHRE-PHASE induction motors are an attractive alternative to their single-phase counterparts in most applications. This is because the three-phase motors, in comparison with single-phase motors, have superior operating characteristics, such as high starting torque, low starting current, high efficiency, better power factor, etc. They are also more economical than their single-phase counterparts. However, in many situations, only a single-phase supply is available, and a typical solution for operating the three-phase motor has been through the use of a single-to-three-phase converter interface. This is particularly true of rural and remote areas, where a single-phase supply line is preferred by the electricity authorities for economical reasons, while the consumers prefer three-phase pumps or motors for their benefit. In a traction application, on the other hand, there is no alternative to a single-phase supply from the traction system. Even if this

Paper IPCSD 97-09, presented at the 1990 Industry Applications Society Annual Meeting, Seattle, WA, October 7-12, and approved for publication in the IEEE Transactions on Industry Applications by the Electric Machines Committee of the IEEE Industry Applications Society. Manuscript released for publication January 13, 1997.

S. K. Biswas is with the Department of Electrical Engineering, Jadavpur University, Calcutta 700 032, India.

C. Chakraborty was with the Department of Electrical Engineering, Indian Institute of Technology, Kharagpur 721302 , India. He is now with the Department of Electrical Engineering, Mie University, Mie, Japan, on leave from the Department of Electrical Engineering, Jadavpur University, Calcutta 700 032, India.

B. Basak was with the Department of Electrical Engineering, Jadavpur University, Calcutta 700 032, India. He is now with the Department of Electrical Engineering, Bengal Engineering College, Howrah 711 103, India.

D. P. Sen Gupta is with the Department of Electrical Engineering, Indian Institute of Science, Bangalore 560 012, India.

Publisher Item Identifier S 0093-9994(98)04923-8.

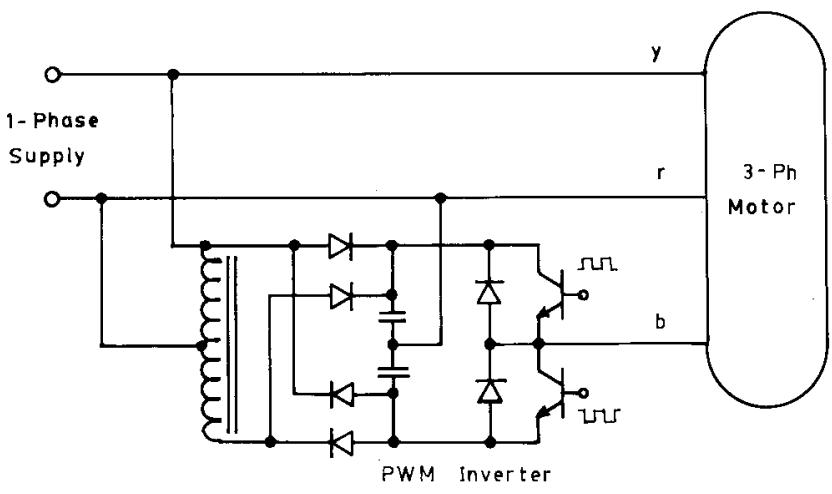

(a)

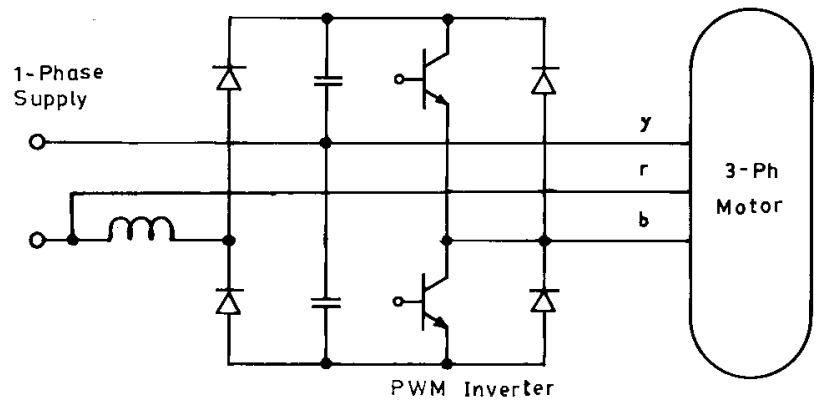

(b)

Fig. 1. (a) Asymmetrical single-to-three-phase converter. (b) Modified asymmetrical phase converter.

is acceptable for the traction motors (which are commonly dc motors operating from rectified supply), the auxiliary equipment, consisting of pumps, fans, and air conditioners, use three-phase motors. Thus, the single-to-three-phase converter is indispensable.

Phase conversion equipment has been designed either with rotary equipment or passive equipment, like capacitors and magnetic circuit, in the past. Static phase converters using controlled power semiconductor devices have now become common, due to lower cost, size, and better performance of the connected motor. Several types of static phase converter circuits have been developed, with varying performance and circuit complexities [1]-[6]. Of these, a new simple static phase converter topology [4] uses a synthesized supply phase, displaced by $120^{\circ}$ from the available single-phase supply, such that the two supplies can be joined together in an open delta connection to feed the three-phase induction motor load. As shown in Fig. 1(a), the synthesized phase is a pulsewidth modulated (PWM) waveform generated by a static single- 


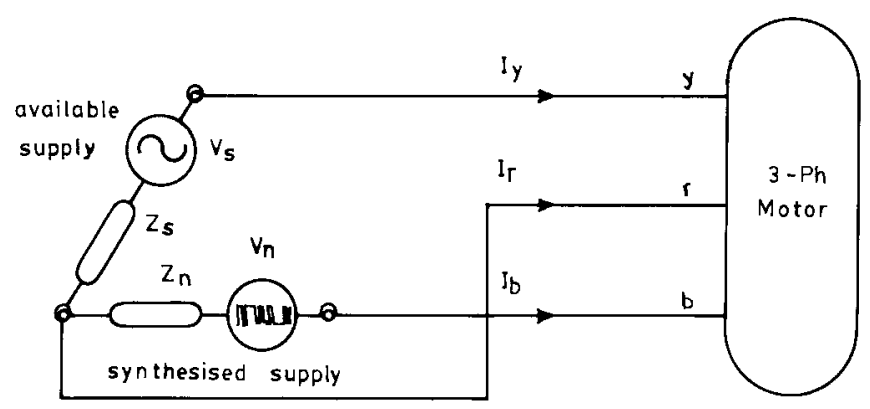

Fig. 2. Simplified model of phase converter.

phase inverter, from the rectified (and filtered) single-phase supply. Thus, the motor has a unique open delta supply system, consisting of a sinusoidal waveform between two lines and a PWM waveform between two other lines (one line being common to both waveforms). The synthesized PWM waveform is selected to have a fundamental equal to that of the sinusoidal supply and minimum magnitude of lower order harmonics. The detailed operation of the phase converter has already been discussed in the literature [4].

A later developed version of the asymmetrical converter using open delta connection utilizes a very simple power circuit with a minimum number of components [6]. As shown in Fig. 1(b), the rectifier stage uses a voltage doubler circuit, which is connected to a half-bridge inverter that is similar to the earlier topology of Fig. 1(a). The elimination of the autotransformer reduces the weight and cost of the system, but the facility of balancing the motor supply or matching the motor to a given supply are lost.

This paper presents an analysis of the performance of an induction motor fed from such an asymmetrical phase converter supply. The analysis has been carried out using the methods of symmetrical components.

\section{SYSTEM MODEL}

In order to obtain a quick understanding of the motor behavior in this case, it is easier to analyze the system under steady state. It is assumed that there is no magnetic saturation and that the motor has perfectly balanced windings without space harmonics. Core loss and other approximately constant losses do not affect torque and, hence, can be modeled by a resistor network across motor terminals, if desired. Skin effect and motor parameter variation with temperature are neglected, and the system is assumed to be linear, such that the superposition theorem can be applied for each harmonic effect. Also, it is assumed that all static switches are ideal, and the rectified dc voltage is constant.

A simplified model of the system is depicted in Fig. 2, which shows the synthesized supply of PWM voltage connected to the available sinusoidal single-phase supply in open delta and supplying power to the three-phase motor load. Here, it is assumed that the fundamental voltage of the synthesized supply is the same in magnitude to that of the available supply, except being phase displaced by $120^{\circ}$. This is to ensure balanced fundamental flux in the machine and reduced torque pulsations. For this purpose, the inverter PWM

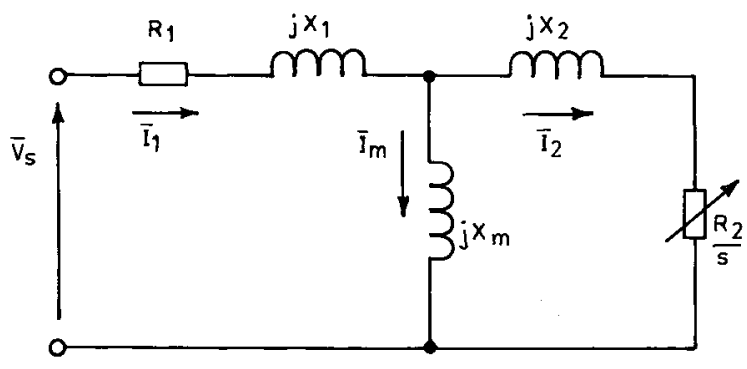

Fig. 3. Per-phase fundamental equivalent circuit.

pattern and the dc-link voltage will have to be fixed (with the help of tappings on the autotransformers, if necessary). Thus, the present system is unique in the sense that it can be resolved into two different supply systems: 1) a balanced three-phase supply of voltage equal to available single-phase supply at fundamental frequency and 2) a single-phase supply of harmonic voltages across one pair of lines (from the synthesized phase). The harmonic voltages, however, will continue to be represented by a single-phase supply, as in the earlier case.

However, if the fundamental voltage of the synthesized phase is different from that of the sinusoidal line, then it generates unbalanced fundamental flux. This condition can be analyzed by representing the system model at fundamental frequency by a balanced three-phase supply system and an additional source of fundamental frequency across one pair of lines (from the synthesized phase). The harmonic voltages, however, will continue to be represented by a single-phase supply, as in the earlier case.

\section{MACHINE ANALYSIS}

\section{A. Fundamental Current Analysis}

As discussed earlier, under balanced fundamental supply, the fundamental quantities constitute a balanced three-phase supply and, hence, the analysis of its effects on the motor can be carried out using the stator-referred per-phase equivalent circuit shown in Fig. 3, as usual. The results can be superimposed to that obtained from harmonic current analysis to get the total effect.

However, if the fundamental supply system is unbalanced, then the system model is first represented by a balanced threephase supply of line-to-line voltage magnitude equal to that of the sinusoidal supply. Since the synthesized phase has an actual fundamental magnitude different from this value, the difference in fundamental is represented by a voltage source across the lines to which the synthesized phase is connected. Thus, the difference in fundamental voltage source can be treated as a separate unbalanced supply system and analyzed in the same way as the harmonics (with $n=1$ ), as discussed in the following section. The final instantaneous current in this case will be obtained by superimposing the results obtained from balanced fundamental analysis, the result from the unbalanced component of fundamental, and the result from harmonic analysis. 


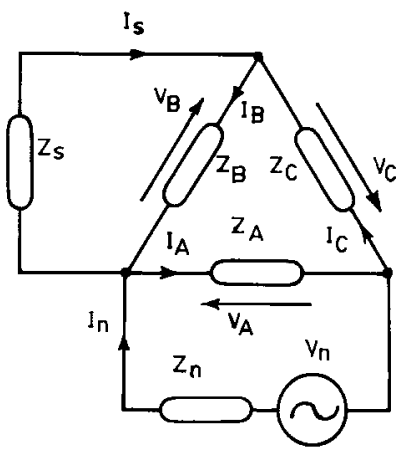

(a)

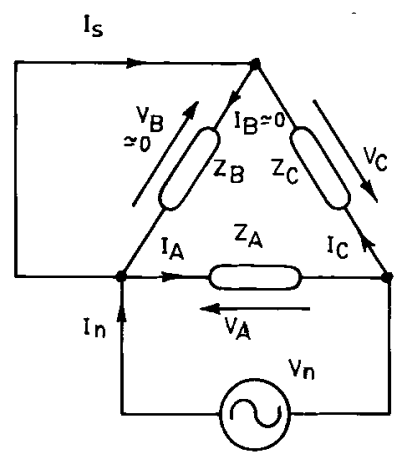

(b)

Fig. 4. Harmonic equivalent circuit of system. (a) With finite source impedance. (b) With zero source impedance.

\section{B. Harmonic Current Analysis}

With regard to the injected harmonics from the synthesized supply, the nature of the problem is a special case of unbalanced operation of an induction motor, since harmonics are injected only across one pair of motor terminals. Different methods of analysis are available in the literature, but the method of symmetrical components [7], [8] is used to simplify the analysis. This permits the resolution of the given unbalanced voltages into positive-, negative-, and zerosequence components, which, when reduced to per-phase values, may be considered to act independently upon each of the balanced phases of the motor. This sets up corresponding sequence current of the $n$th harmonic order, which, when superimposed together, generates the resulting $n$th harmonic current through each motor phase. Finally, all the individual components of harmonic currents and fundamental current of each phase can be superimposed with regard to instantaneous values to obtain the instantaneous current of each phase of the motor.

Since most of the motors are run in delta connection, the case of a delta-connected motor is selected arbitrarily for analysis of the harmonic current generated from the PWM supply, the harmonic voltage content of which is known. Since the superposition theorem is being applied, the harmonic equivalent circuit of the system will be as depicted in Fig. 4(a) with finite source impedances. If a star-connected motor is selected, the analysis will proceed on a similar path.

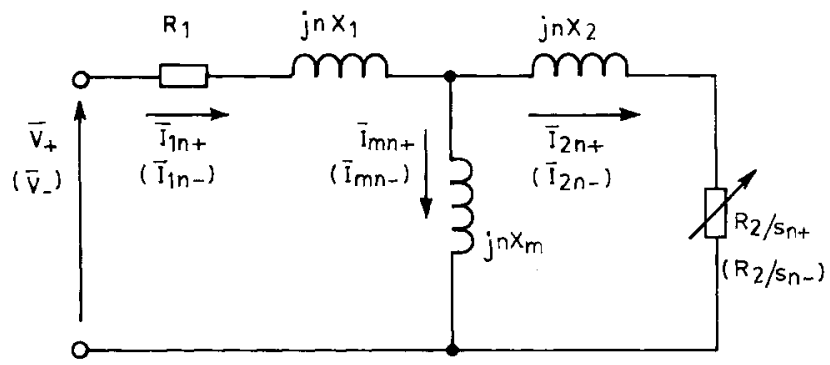

Fig. 5. Per-phase harmonic equivalent circuit for sequence components.

With respect to Fig. 4(a), the following equations can be written:

$$
\begin{aligned}
I_{S} & =I_{B}-I_{C} \\
I_{n} & =I_{A}-I_{C} \\
V_{A}+V_{B}+V_{C} & =0 \\
I_{S} & =-V_{B} Y_{S} \\
V_{A} & =V_{n}-I_{n} Z_{n} \\
V_{A} & =V_{\mathrm{o}}+V_{+}+V_{-} \\
V_{B} & =V_{\mathrm{o}}+a^{2} V_{+}+a V_{-} \\
V_{C} & =V_{\mathrm{o}}+a V_{+}+a^{2} V_{-} \\
I_{A} & =Y_{\mathrm{o}} V_{\mathrm{o}}+Y_{+} V_{+}+Y_{-} V_{-} \\
I_{B} & =Y_{\mathrm{o}} V_{\mathrm{o}}+a^{2} Y_{+} V_{+}+a Y_{-} V_{-} \\
I_{C} & =Y_{\mathrm{o}} V_{\mathrm{o}}+a Y_{+} V_{+}+a^{2} Y_{-} V_{-}
\end{aligned}
$$

where $\mathrm{o},+$, and - are the symbols for zero, positive, and negative components, respectively, and " $a$ " is the operator, as usual, of symmetrical component analysis. $Y_{S}$ and $Y_{n}$ are the reciprocals of $Z_{s}$ and $Z_{n}$, respectively, while $Y_{\mathrm{o}}, Y_{+}$, and $Y_{-}$ are the motor sequence admittances. Substituting (6)-(8) in (3),

$$
V_{\mathrm{o}}=0 \text {. }
$$

Substituting (4) in (1) and simplifying,

$$
V_{B}=\left(I_{C}-I_{B}\right) Z_{S}
$$

Substituting (7), (10), and (11) in (13) and simplifying,

$$
\left(j \sqrt{3} Z_{S} Y_{+}-a^{2}\right) V_{+}=\left(a+j \sqrt{3} Z_{S} Y_{-}\right) V_{-} .
$$

Substituting (2) in (5),

$$
V_{A}=V_{n}+\left(I_{C}-I_{A}\right) Z_{n}
$$

Substituting (6), (9), and (11) in (15) and simplifying,

$$
V_{+}\left[1+(1-a) Z_{n} Y_{+}\right]+V_{-}\left[1+\left(1-a^{2}\right) Z_{n} Y_{-}\right]=V_{n}
$$

Solving (14) and (16),

$$
\begin{aligned}
& V_{+}=\frac{V_{n}\left(3 Z_{S} Y_{-}-j \sqrt{3} a\right)}{3\left[1+\left(Z_{S}+Z_{n}\right)\left(Y_{+}+Y_{-}\right)+3 Z_{S} Z_{n} Y_{+} Y_{-}\right]} \\
& V_{-}=\frac{V_{n}\left(3 Z_{S} Y_{+}+j \sqrt{3} a^{2}\right)}{3\left[1+\left(Z_{S}+Z_{n}\right)\left(Y_{+}+Y_{-}\right)+3 Z_{S} Z_{n} Y_{+} Y_{-}\right]} .
\end{aligned}
$$

In practice, the source impedances may be neglected, particularly for the synthesized phase (due to the presence of a stiff 


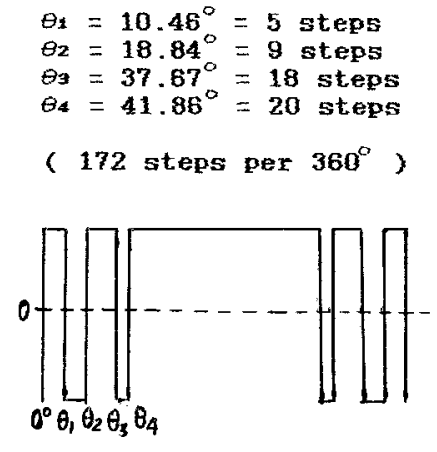

Fig. 6. PWM pattern used and harmonic content.

dc bus of the inverter). On the other hand, the effect of source impedance is to change the distribution of harmonic current flow through the motor windings that appear in parallel to them in the equivalent circuit. The lower order harmonics are kept low by the careful selection of the PWM pattern. Thus, any changes in the current distribution by neglecting the source impedance will not reflect on the actual motor current to a significant level. At higher harmonics, both source and motor relative current distribution does not change significantly, while the higher impedances keep the higher harmonic currents to low values. Thus, the source impedances can be neglected to simplify the analysis, without a major change in the overall superimposed current pattern. In this case, the symmetrical components of harmonic voltages are obtained as

$$
\begin{aligned}
& V_{+}=\frac{V_{n}}{\sqrt{3}} \angle \pi / 6 \\
& V_{-}=\frac{V_{n}}{\sqrt{3}} \angle-\pi / 6 .
\end{aligned}
$$

Note that the zero-sequence component is absent, as per (12). The equivalent circuit in this case is shown in Fig. 4(b), where it is clear that no harmonic current will flow through $Z_{B}$, since it is shorted by the presence of the available supply without source impedance. Even if source impedance is considered, its value will be less than the motor phase impedance and, hence, only a small current can flow through $Z_{B}$. So far as any of these sequence components is concerned, the motor will behave the same way as if a balance voltage is applied across its three line terminals. This permits the analysis to be carried out using corresponding per-phase steady-state equivalent circuits. The harmonic equivalent circuit for positive- and negative-sequence components will not be the same, since the slip frequency has to be considered according to the direction of the rotation of the corresponding flux components. Thus,

$$
\begin{aligned}
& S_{n+}=1-(1-s) / n \\
& S_{n-}=1+(1-s) / n .
\end{aligned}
$$

In the general case, the nth-order harmonic voltage can be written from (19) and (20) in terms of its positive- and negative-sequence components with phase angle $\phi_{n}$ as

$$
\begin{aligned}
& V_{n+}=\frac{V_{n}}{\sqrt{3}} \angle\left(\phi_{n}+\pi / 6\right) \\
& V_{n-}=\frac{V_{n}}{\sqrt{3}} \angle\left(\phi_{n}-\pi / 6\right) .
\end{aligned}
$$

\begin{tabular}{cc} 
Order of & Magnitude \% of \\
Harmonic & De link value \\
\hline 1 & 106.00390 \\
3 & 0.59129 \\
5 & 3.43444 \\
7 & 1.62575 \\
9 & 10.96111 \\
11 & 16.89074 \\
13 & 8.55569 \\
15 & 42.56456
\end{tabular}

The $n$ th-order harmonic equivalent circuit of the induction motor to corresponding positive- and negative-sequence harmonic voltage is shown in Fig. 5. The instantaneous-sequence component currents for each $n$ th-order harmonic are calculated using the corresponding harmonic equivalent circuit.

\section{Phase and Line Currents}

Continuing the assumption of a delta-connected motor, the motor instantaneous phase currents can be obtained by superposing the results of fundamental analysis and harmonic analysis. Considering the approximate equivalent circuit of Fig. 3, the actual phase currents can be written as

$$
\begin{aligned}
i_{A}= & I_{P 1} \cos \left(\omega t+\theta_{1}\right)+\sum_{n=3}^{N}\left[I_{P n+} \cos \left(n \omega t+\theta_{n+}\right)\right. \\
& \left.+I_{P n-} \cos \left(n \omega t+\theta_{n-}\right)\right] \\
i_{B}= & I_{P 1} \cos \left(\omega t+\theta_{1}-2 \pi / 3\right) \\
& +\sum_{n=3}^{N}\left[I_{P n+} \cos \left(n \omega t+\theta_{n+}-2 \pi / 3\right)\right. \\
& \left.+I_{P n-} \cos \left(n \omega t+\theta_{n-}+2 \pi / 3\right)\right] \\
i_{C}= & I_{P 1} \cos \left(\omega t+\theta_{1}+2 \pi / 3\right) \\
& +\sum_{n=3}^{N}\left[I_{P n+} \cos \left(n \omega t+\theta_{n+}+2 \pi / 3\right)\right. \\
& \left.+I_{P n-} \cos \left(n \omega t+\theta_{n-}-2 \pi / 3\right)\right]
\end{aligned}
$$

where $n=3,5,7, \cdots, N$.

$I_{P 1}$ and $I_{P n}$ are the peak fundamental and peak $n$th harmonic currents, respectively, along with its phase angle $\theta_{1}$ or $\theta_{n}$, respectively. The line currents $i_{r}, i_{y}$, and $i_{b}$ can be calculated from the phase currents as

$$
\begin{aligned}
& i_{r}=i_{A}-i_{B} \\
& i_{y}=i_{B}-i_{C} \\
& i_{b}=i_{C}-i_{A} .
\end{aligned}
$$

\section{Torque Computation}

The instantaneous torque can be easily expressed in terms of the corresponding two phase current quantities [9] instead of the actual three phase quantities. Thus, the torque is given by

$$
m=(3 / 2) p\left(\psi_{1 \alpha} i_{1 \beta}-\psi_{1 \beta} i_{1 \alpha}\right) .
$$




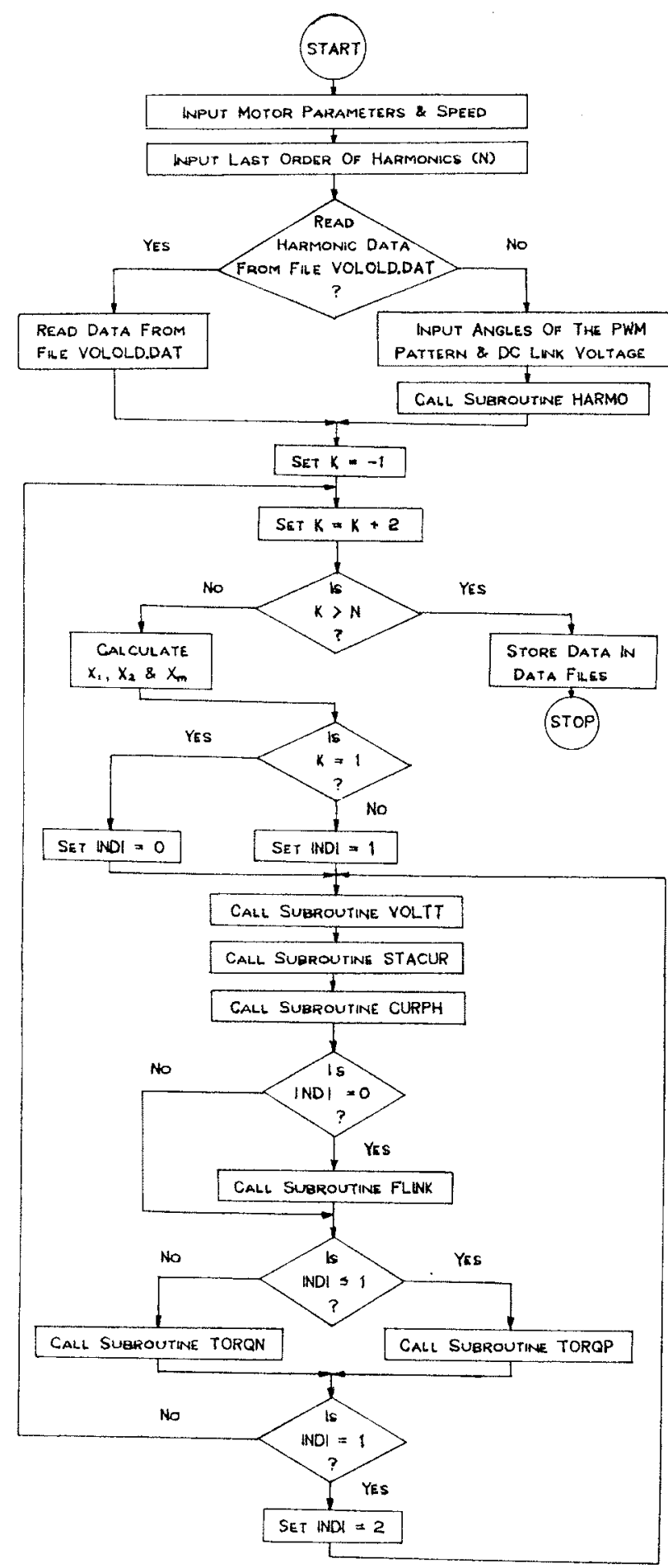

Fig. 7. Flow chart of the computer program.

It can also be shown [9] that

$$
\begin{aligned}
& \psi_{1 \alpha}=\int\left(v_{1 \alpha}-R_{1} i_{1 \alpha}\right) d t \\
& \psi_{1 \beta}=\int\left(v_{1 \beta}-R_{1} i_{1 \beta}\right) d t \\
& v_{1 \alpha}=v_{A} \\
& v_{1 \beta}=\left(v_{B}-v_{C}\right) / \sqrt{3} \\
& i_{1 \alpha}=i_{A} \\
& i_{1 \beta}=\left(i_{B}-i_{C}\right) / \sqrt{3}
\end{aligned}
$$

where $\alpha$ and $\beta$ are the equivalent two phase quantities of the actual three phase variables $A, B$, and $C$, and $p$ is the number of pole pairs.

The balanced fundamental current flow will result in the development of a steady torque in the motor. The presence of harmonic currents will, however, result in the development of two types of harmonic torques: 1) a steady torque due to the interaction of a harmonic current with harmonic flux of the same order and 2) an oscillatory torque (with zero average value) due to the interaction of a harmonic current with harmonic flux of different order. The steady harmonic torques will be negligible due to two reasons: 1) harmonic currents and harmonic flux will both be of relatively small magnitudes compared to fundamental, while 2) the forward torque produced by positive sequence components will be partially canceled by the reverse torque produced by negativesequence components. The oscillatory torque produced by the interaction of harmonic currents with the fundamental flux (the most significant flux in the machine) will be most predominant and, hence, this is the only torque pulsation being considered for analysis here. Thus, the general torque equation for the motor can be written as

$$
\begin{aligned}
m= & (3 / 2) p\left(\psi_{1 \alpha} i_{1 \beta}-\psi_{1 \beta} i_{1 \alpha}\right) \\
& +(3 / 2) p \sum_{n=3}^{N}\left[\psi_{1 \alpha}\left(i_{1 \beta n+}+i_{1 \beta n-}\right)\right. \\
& \left.-\psi_{1 \beta}\left(i_{i \alpha n+}+i_{1 \alpha n-}\right)\right]
\end{aligned}
$$

In order to solve for the developed torque, first consider the balanced fundamental three phase quantities only:

$$
\begin{aligned}
& v_{A}=V_{P} \cos \omega t \\
& v_{B}=V_{P} \cos (\omega t-2 \pi / 3) \\
& v_{C}=V_{P} \cos (\omega t+2 \pi / 3) \\
& i_{A}=I_{P} \cos \left(\omega t+\theta_{1}\right) \\
& i_{B}=I_{P} \cos \left(\omega t-2 \pi / 3+\theta_{1}\right) \\
& i_{C}=I_{P} \cos \left(\omega t+2 \pi / 3+\theta_{1}\right) .
\end{aligned}
$$

Substituting these expressions in (32)-(37) and solving,

$$
\begin{aligned}
i_{1 \alpha} & =I_{P} \cos \left(\omega t+\theta_{1}\right) \\
i_{1 \beta} & =I_{P} \cos \left(\omega t-\pi / 2+\theta_{1}\right) \\
\psi_{1 \alpha} & =\psi_{P 1 \alpha} \sin (\omega t+\delta) \\
\psi_{1 \beta} & =\psi_{P 1 \beta} \sin (\omega t-\pi / 2+\delta) .
\end{aligned}
$$

The positive-sequence components of harmonics also form a balanced three-phase supply, as given by

$$
\begin{aligned}
& v_{A n+}=V_{P n+} \cos \left(n \omega t+\pi / 6+\phi_{n}\right) \\
& v_{B n+}=V_{P n+} \cos \left(n \omega t-\pi / 2+\phi_{n}\right) \\
& v_{C n+}=V_{P n+} \cos \left(n \omega t+5 \pi / 6+\phi_{n}\right) .
\end{aligned}
$$

Proceeding as in the case of the fundamental, the equivalent two phase currents can be expressed as

$$
\begin{aligned}
& i_{1 \alpha n+}=I_{P n+} \cos \left(n \omega t+\theta_{n+}\right) \\
& i_{1 \beta n+}=I_{P n+} \cos \left(n \omega t-\pi / 2+\theta_{n+}\right) .
\end{aligned}
$$




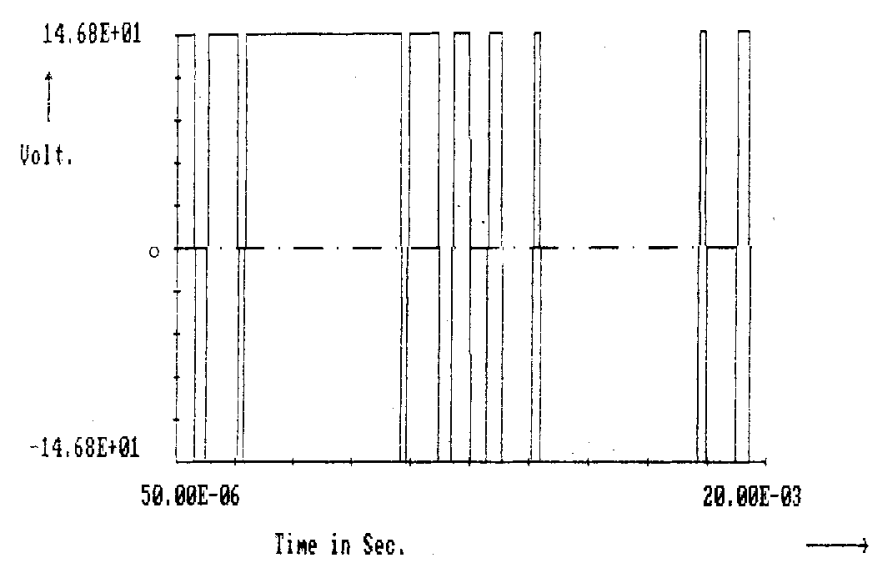

(a)

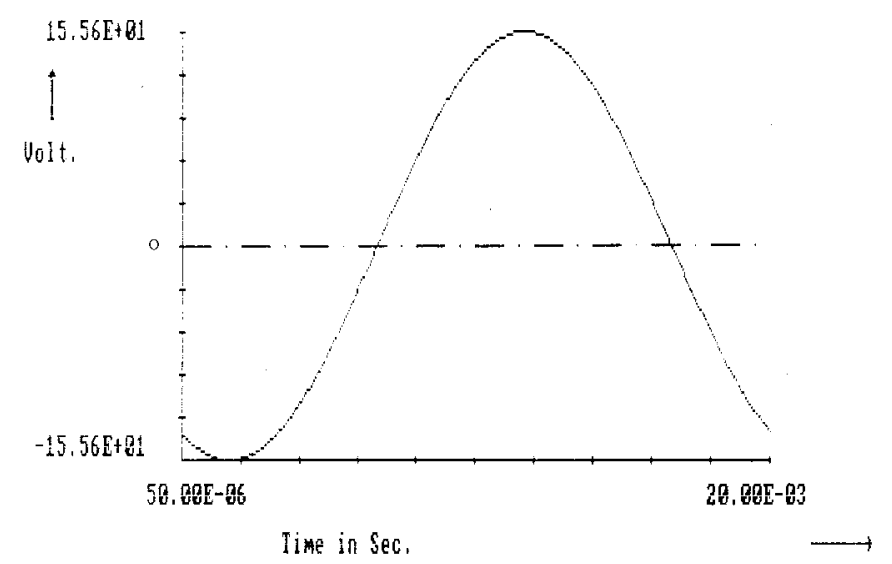

(b)

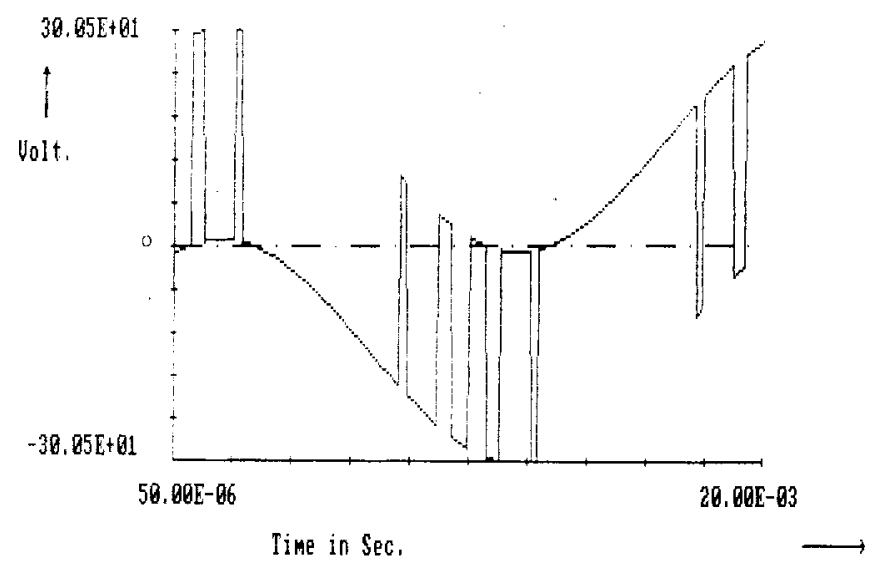

(c)

Fig. 8. Simulated line voltages of the motor. (a) Voltage across line B and $\mathrm{R}\left(V_{\text {peak }}=146.8 \mathrm{~V}\right)$. (b) Voltage across line $\mathrm{R}$ and $\mathrm{Y}\left(V_{\text {peak }}=155.6\right.$ V). (c) Voltage across line $\mathrm{Y}$ and $\mathrm{B}\left(V_{\text {peak }}=300.5 \mathrm{~V}\right)$.

The negative-sequence components of harmonic voltage which form a balanced three-phase supply can be expressed as

$$
\begin{aligned}
& v_{A n-}=V_{P n-} \cos \left(n \omega t-\pi / 6+\phi_{n}\right) \\
& v_{B n-}=V_{P n-} \cos \left(n \omega t+\pi / 2+\phi_{n}\right) \\
& v_{C n-}=V_{P n-} \cos \left(n \omega t-5 \pi / 6+\phi_{n}\right) .
\end{aligned}
$$

Proceeding as earlier, the equivalent two phase currents can be expressed as

$$
\begin{aligned}
& i_{1 \alpha n-}=I_{P n-} \cos \left(n \omega t+\theta_{n-}\right) \\
& i_{1 \beta n-}=I_{P n-} \cos \left(n \omega t+\pi / 2+\theta_{n-}\right) .
\end{aligned}
$$

The instantaneous value of torque can be obtained by substituting (45)-(48), (52), (53), (57), and (58) in (38).

\section{Simulation}

In order to verify the mathematical analysis, the system was simulated on a digital computer, so as to obtain instantaneous waveforms of line-to-line voltage, line currents, and torque as per the analysis procedure. A fixed PWM pattern was selected for this purpose (as shown in Fig. 6), such that it has reduced low-order harmonics without involving high- frequency switching. The PWM steps occur at angles $10.46^{\circ}$, $18.84^{\circ}, 37.67^{\circ}$, and $41.86^{\circ}$, respectively, in the first quarter cycle and symmetrically thereafter. This has resulted in a fundamental peak of $106 \%$ of the dc-link voltage and less than $3.5 \%$ of harmonics up to the seventh. For the purpose of simulation, harmonics up to the 101st was considered to be adequate, since higher harmonics have negligible effect. A 1-hp motor, available in the laboratory, was selected for use in verification of analytical results. The motor equivalent circuit parameters were measured and used in the analysis. The motor was connected in delta to be operated from a $110-\mathrm{V}$ line voltage. Thus, the sinusoidal voltage supply was selected to be $110 \mathrm{~V}$-rms, while the dc-link voltage was adjusted to $293.5 \mathrm{~V}$, so that the PWM waveform generates a fundamental of rms value $110 \mathrm{~V}$ through the half-bridge inverter. All these conditions were assumed for simulation and actually maintained during experimentation. The flow chart for the computer program developed for such purposes [10], [11] is given in Fig. 7. The computation is carried out with an efficient handling of several files and subroutines [10], [11]. Subroutines, such as HARMO (to find different harmonic voltages corresponding to a PWM pattern), VOLTT (to find the positive- and negative-sequence harmonic voltage, reactances, and modified rotor resistances owing to different 


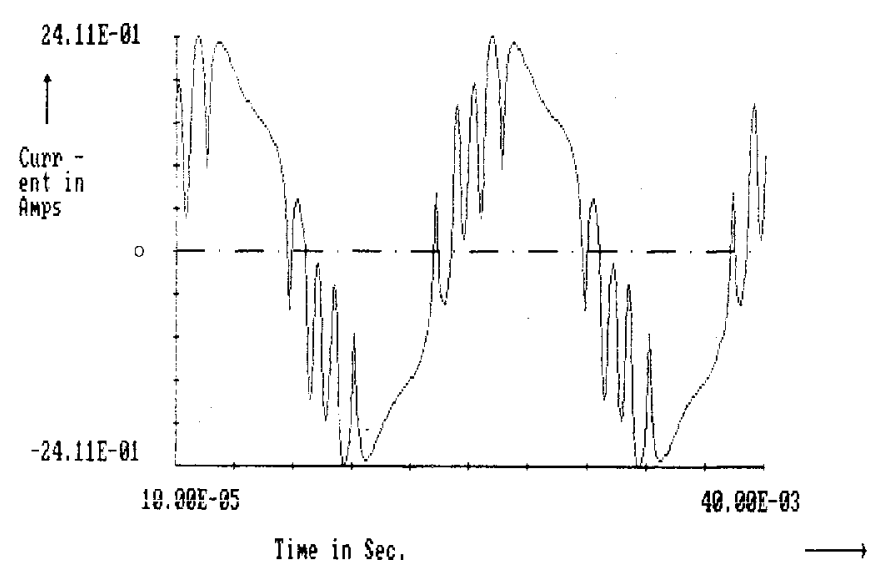

(a)

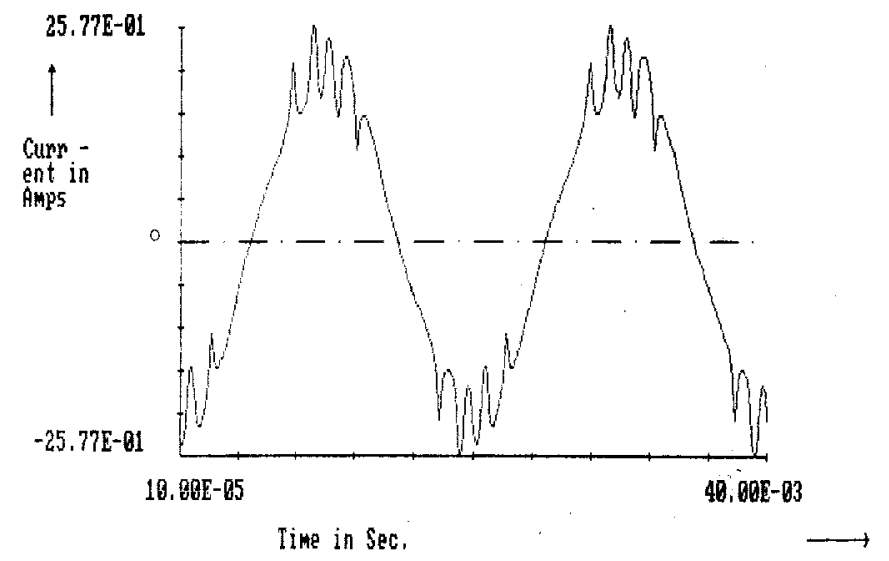

(b)

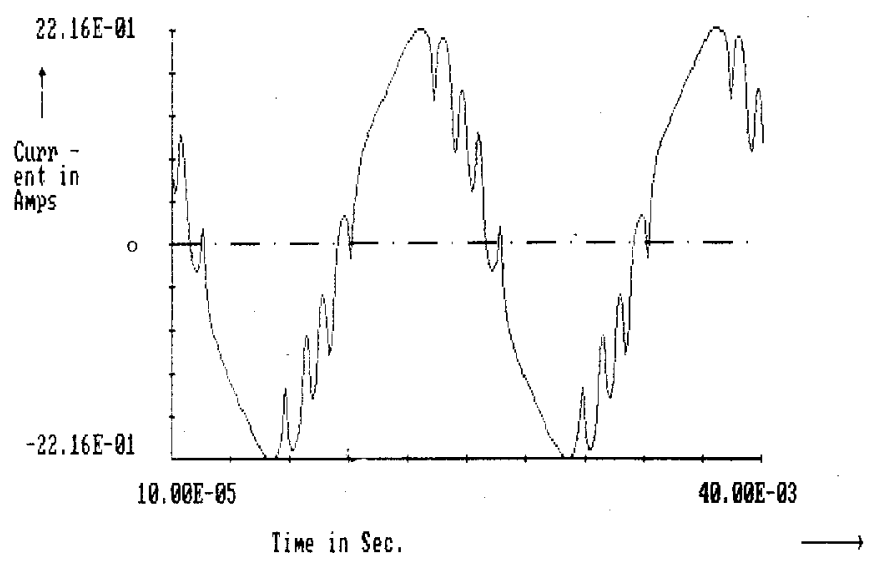

(c)

Fig. 9. Simulated line currents of motor. (a) Current in line $\mathrm{R}\left(I_{\text {peak }}=2.41 \mathrm{~A}\right)$. (b) Current in line $\mathrm{Y}\left(I_{\text {peak }}=2.58\right.$ A). (c) Current in line $\mathrm{B}\left(I_{\text {peak }}=2.22 \mathrm{~A}\right)$.

magnitudes of slips), STACUR (to compute the $R$-phase current in complex or phasor form), CURPH (to find the nature of all instantaneous three phase currents, storing them in files to be plotted when necessary), FLINK (to compute flux linkages, e.g., $\psi_{1 \alpha}$ and $\psi_{1 \beta}$ ), TORQP (to compute torque owing to positive-sequence currents), and TORQN (to estimate torque owing to negative-sequence currents) are invoked in proper places in the main program, as shown in the flow chart. $K$, which represents the order of harmonic to be considered is incremented by 2 in subsequent steps to account for the effect of odd harmonic order (the even order being absent), thus speeding up the execution. An indicator "INDI" is set to " 0 " for fundamental, " 1 " for positive-sequence, and " 2 " for negative-sequence calculations in the program. In different cases, for example, in order to compute torque, current, etc., the status of "INDI" is tested first and, accordingly, program control is directed to corresponding structure (i.e., group of equations). Initially, "INDI" is set to 0 for computations of fundamental quantities, and then, for every higher harmonic order, "INDI" assumes " 1 " to compute all positive-sequence quantities and " 2 " for negative-sequence quantities. Calculations continue until $K$ (the running order of harmonics) exceeds $N$ (the maximum order to be taken into account). For the present study, up to 101st harmonic order is considered.

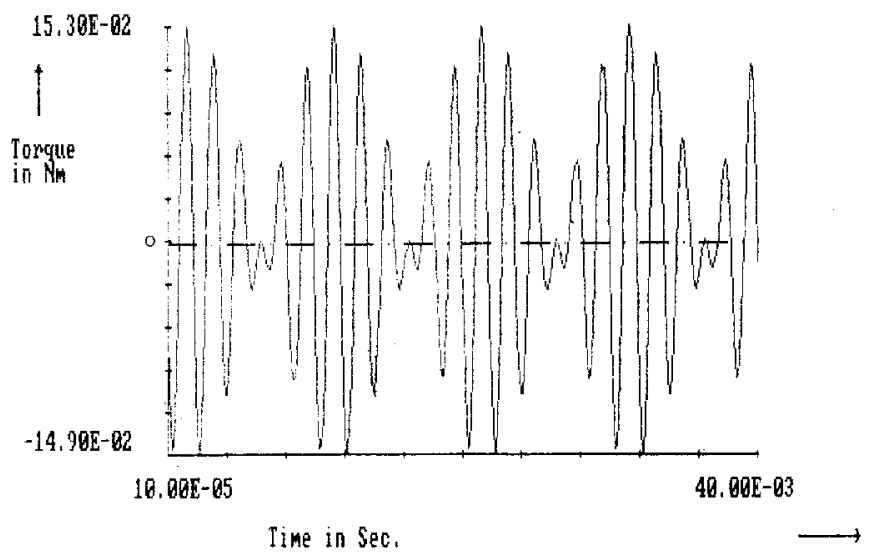

Fig. 10. Simulated torque due to the interaction of 11th harmonic current with fundamental flux (peak $=0.153 \mathrm{~N} \cdot \mathrm{m}$ ).

The resultant torque pattern is stored in the file RTOR. DAT, which is created by summing up all positive- and negativesequence torque up to the 101st order of harmonics. The individual torque nature can be had from the file TOR.DAT.

The simulated results are depicted in Figs. 8-11. The impressed line-to-line voltages are shown in Fig. 8, while the resulting line current simulation is shown in Fig. 9. A 


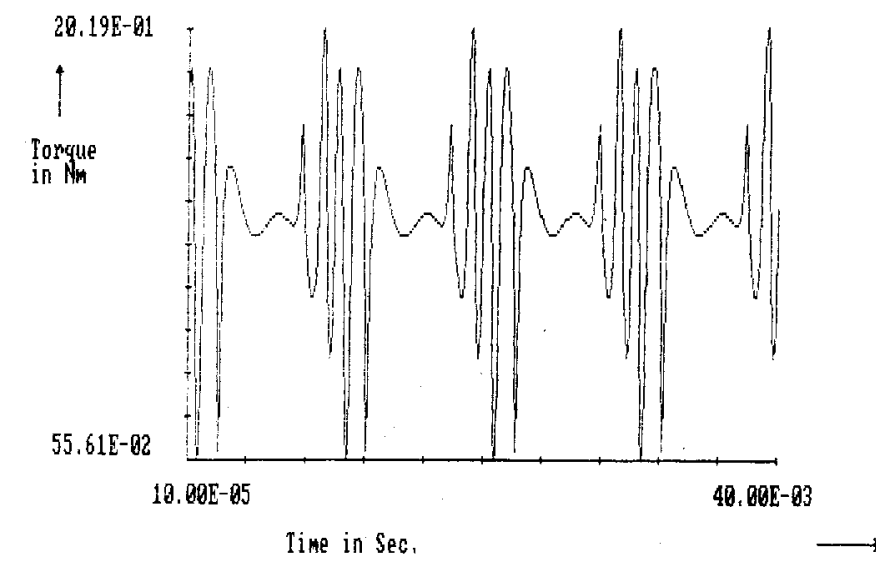

Fig. 11. Simulated resultant torque (peak $=2.02 \mathrm{~N} \cdot \mathrm{m})$.

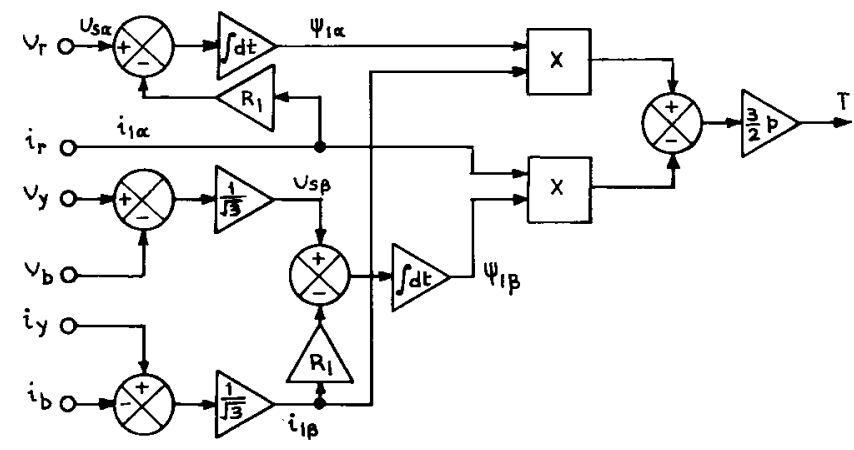

Fig. 12. Analog torque computation circuit.

sample torque pattern produced due to the interaction of eleventh harmonic current with fundamental flux is shown in Fig. 10. The simulated pattern of the developed torque is shown in Fig. 11 for the condition of a partially loaded motor.

\section{EXPERIMENTAL RESULTS}

An experimental setup was made to verify the simulated results under identical conditions. The converter topology shown in Fig. 1(a) was used, such that the fundamental voltage to the motor can be balanced through the transformer tappings [4]. The inverter was made using bipolar power transistors and the PWM pattern selected (Fig. 6) was stored in an electrically programmable read-only memory (EPROM) in the control logic. The EPROM output was phase locked at an angle of $60^{\circ}$ (i.e., $120^{\circ}$ lead) with respect to the supply line, such that the synthesized phase output creates an open delta connection with the incoming supply. A variac was connected to the incoming single-phase line and the voltage was gradually built up through the variac to overcome high starting currents, which would have otherwise required a separate starting arrangement [4].

An analog computation circuit was used [9] to calculate the torque developed by the motor, as shown in Fig. 12. This utilizes the actual instantaneous line currents and voltages of the motor for the torque calculation, using the equivalent circuit parameters.

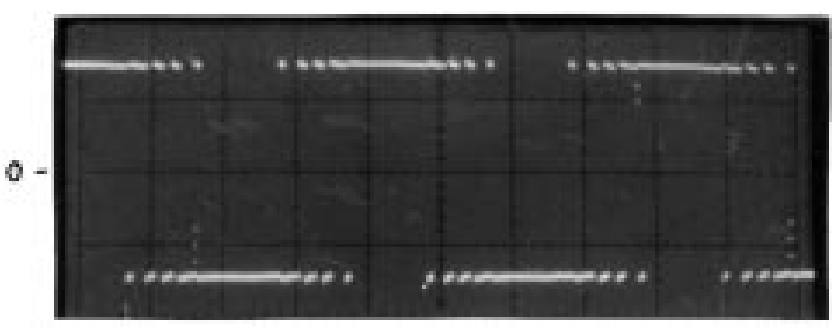

(a)

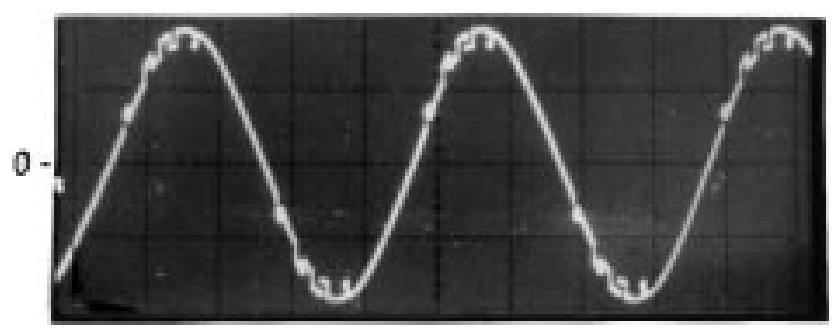

(b)

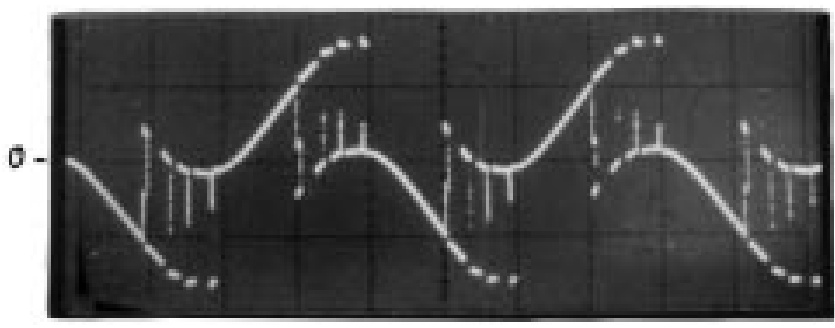

(c)

Fig. 13. Experimental line voltages of motor. (a) Voltage across line B and $\mathrm{R}(100 \mathrm{~V} / \mathrm{div})$. (b) Voltage across line R and Y (100 V/div). (c) Voltage across line $\mathrm{Y}$ and $\mathrm{B}(200 \mathrm{~V} / \mathrm{div})($ time $=5 \mathrm{~ms} / \mathrm{div})$.

The experimental oscillograms are shown in Figs. 13-15. Fig. 13 shows the three line voltage waveforms of the motor, while Fig. 14 shows the three line currents. The computed torque waveform is shown in Fig. 15. The close similarity between the simulated and experimental waveforms proves the validity of the analysis. The difference noticed can be contributed to the following:

1) the actual machine not being a perfectly linear system;

2) no-load loss component of current being neglected in the simulation;

3) consideration of harmonics only up to the 101st order;

4) finite source impedances.

\section{CONCLUSION}

This paper has presented the analysis of the line current and developed torque of a three-phase motor when fed from a unique asymmetrical supply from a type of single-to-threephase static converter. The system, consisting of one sinusoidal supply and a PWM supply connected in open delta to the motor, has been shown to be equivalent to a balanced threephase supply at fundamental frequency and a set of balanced positive- and negative-sequence components of harmonics. Thus, the individual components of current can be calculated using a per-phase equivalent circuit and the results superim- 


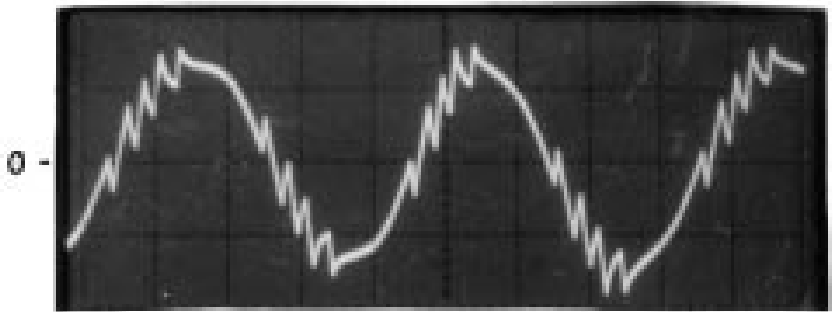

(a)

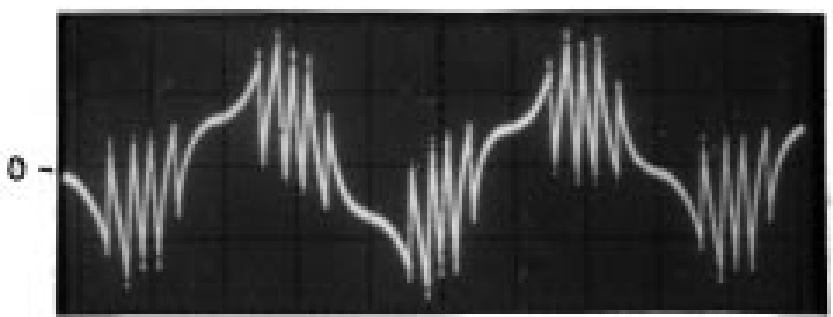

(b)

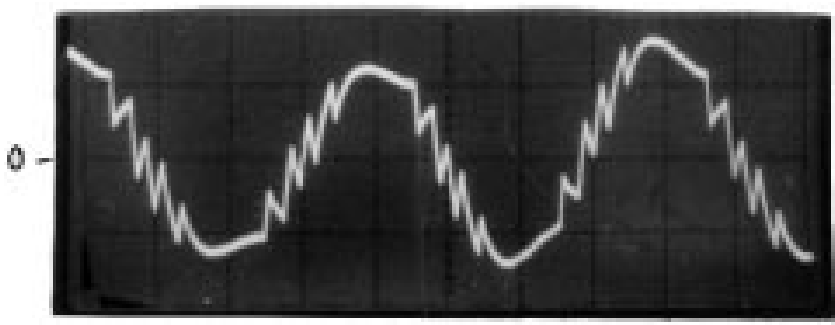

(c)

Fig. 14. Experimental line currents of motor. (a) Current in line R (2 A/div) (b) Current in line $\mathrm{Y}(2 \mathrm{~A} / \mathrm{div})$. (c) Current in line $\mathrm{B}(2 \mathrm{~A} / \mathrm{div})$ (time $=5$ $\mathrm{ms} / \mathrm{div})$.

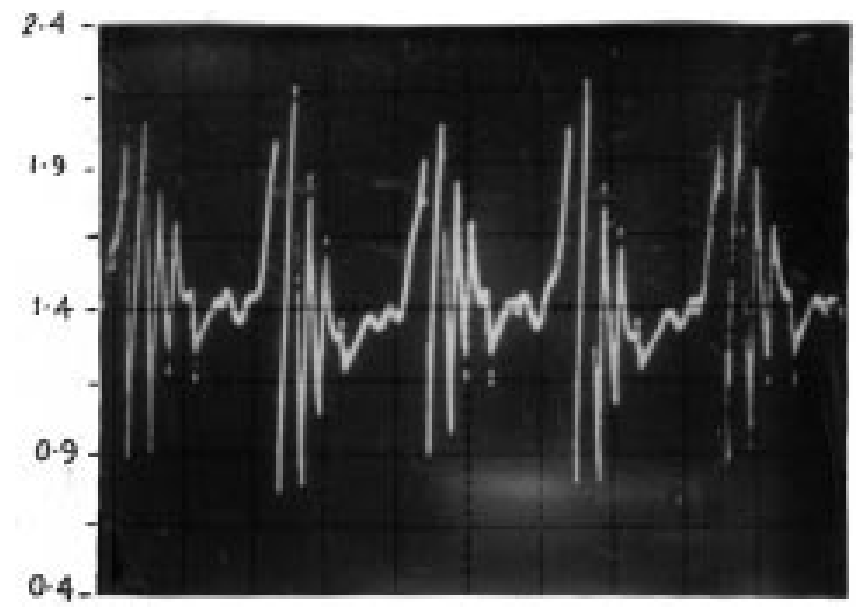

Fig. 15. Resultant torque oscillogram (torque $=0.25 \mathrm{~N} \cdot \mathrm{m} / \mathrm{div}$ ) (time $=5$ $\mathrm{ms} / \mathrm{div})$.

posed to obtain the resulting current. The developed torque has been shown to be consisting of a steady value due to a fundamental component and an oscillating component due to harmonic, resulting in torque pulsations. The exact nature of pulsation, however, depends upon the amount of harmonics, i.e., on the exact PWM pattern. The simulation results based on the mathematical analysis are found to be in fairly close agreement with the actual waveforms, confirming the validity of the analysis. During simulation, it was assumed that source impedances are negligible and the fundamental supply is balanced. However, the analysis under conditions of finite source impedance and unbalanced fundamental supply have also been discussed.

\section{REFERENCES}

[1] K. Frank and K. Rehnman, "Static frequency converters for the auxiliary power supply on electric locomotives and multiple unit trains," ASEA J., vol. 54, no. 5/6, pp. 137-142, 1981.

[2] S. B. Dewan and M. Showleh, "A novel static single to three phase converter," IEEE Trans. Magn., vol. MAG-17, pp. 3287-3289, Nov. 1981.

[3] P. G. Holmes, "Single to 3-phase transient phase conversion in induction motor drives," Proc. Inst. Elect. Eng., vol. 132, pt. B, no. 5, pp. 289-296, Sept. 1985.

[4] S. K. Biswas, "A new static converter for the operation of three-phase motors on single-phase supply," in Conf. Rec. IEEE-IAS Annu. Meeting, 1986, pp. 1550-1554.

[5] S. I. Khan, P. D. Ziogas, and M. H. Rashid, "A novel single to three phase static converter," in Conf. Rec. IEEE-IAS Annu. Meeting, 1987, pp. 658-665

[6] C. Chen, D. M. Divan, and D. W. Novotny, "A single phase to three phase power converter for motor drive applications," in Conf. Rec. IEEE-IAS Annu. Meeting, 1992, pp. 639-646.

[7] J. E. Brown and O. I. Butler, "A general method of analysis of threephase induction motors with asymmetrical primary connections," Proc. Inst. Elect. Eng., vol. 100, pt. II, pp. 25-34, Feb. 1953.

[8] C. F. Wagner and R. D. Evans, Symmetrical Components. New York: McGraw-Hill, 1933.

[9] S. K. Biswas, S. Sathiakumar, and J. Vithayathil, "High efficiency direct torque control scheme for a CSI fed induction motor drive," in Conf. Rec. IEEE-IAS Annu. Meeting, 1986, pp. 216-221.

[10] C. Chakraborty, "Induction motor fed from an asymmetrical phase converter," M.E.E. thesis, Elect. Eng. Dep., Jadavpur Univ., Calcutta, India, 1989

[11] C. Chakraborty, S. N. Bhadra, A. K. Chattopadhyay, and S. K. Biswas, "Digital computation of harmonic torques in an induction motor fed from a static 1-phase to 3-phase converter," J. Inst. Eng. (India), vol. EL-74, pp. 126-131, Nov. 1993.

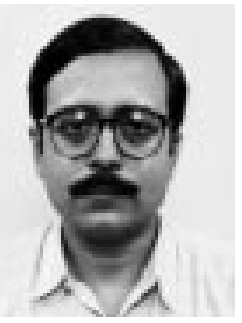

Sujit Kumar Biswas (S'87-M'87-SM'91) received the B.E.E. degree (with honors) from Jadavpur University, Calcutta, India, and the M.E. degree (with distinction) and the Ph.D. degree from the Indian Institute of Science, Bangalore, India, in 1978, 1980, and 1986, respectively, all in electrical engineering.

In July 1987, he joined the faculty of the Department of Electrical Engineering, Jadavpur University, as a Reader. He is currently a Professor. His fields of interest are static power conversion, electrical drives, power semiconductor applications, magnetics, and applied electronics. He has authored numerous research publications, which have been published in India and abroad, in addition to circuit-ideas/popular-electronics articles. He also holds two patents on semiconductor power converter circuits and has been a consultant to several industries.

Dr. Biswas received the Jagadis Bose National Science Talent Search Scholarship in 1974, the Gold Medal of the Institution of Engineers (India) in 1986, the Indian National Science Academy Medal for Young Scientists in 1987, the Certificate of Merit of the Institution of Engineers (India) in 1988, the Pandit M. M. Malaviya Memorial Gold Medal in 1989, and the Tata Rao Prize in 1994. His biography is listed in Reference India, a collection of biographical notes about men and women of achievement. He is a member of the Institution of Engineers (India), a Fellow of the Institution of Electronics and Telecommunication Engineers (India), a life member of the Solar Energy Society of India, and a senior member of the IEEE Industry Applications (IAS), IEEE Industrial Electronics, and IEEE Power Electronics Societies. He is also a member of the IAS Industrial Power Converter Committee. 


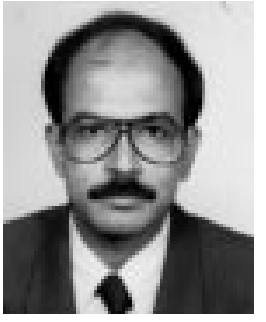

Chandan Chakraborty was born in Calcutta, India. He received the B.E.E. degree (with honors) and the M.E.E. degree from the Department of Electrical Engineering, Jadavpur University, Calcutta, India, in 1987 and 1989, respectively, and the Ph.D. degree from the Indian Institute of Technology, Kharagpur, India, in 1997.

Since 1993, he has been a Lecturer in the Department of Electrical Engineering, Jadavpur University. He was awarded the Japanese Government Scholarship in 1997 for Post-Doctoral studies in Japan, for a two-year period, and is currently with the Department of Electrical Engineering, Mie University, Mie, Japan. His fields of interest are static power conversion, electric drives, and induction generators. He has authored several research papers.

Dr. Chakraborty is a recipient of the Tata Rao Prize of the Institution of Engineers (India)

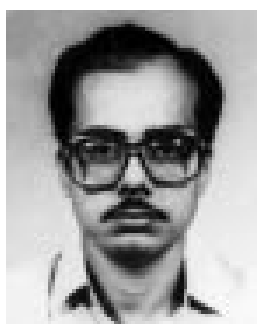

Biswarup Basak (M'92) received the B.E.E. degree (with honors) from the Department of Electrical Engineering, Jadavpur University, Calcutta, India, in 1978 and the M.Sc.(Eng.) degree from the Department of Electrical Engineering, Indian Institute of Science, Bangalore, India, in 1985.

He was with the General Electric Company of India Ltd., Calcutta, India, from 1978 to 1982. He joined Bentec Electricals and Electronics Pvt. Ltd., Calcutta, India, in 1985 and acted as Chief Engineer for one year. In 1987, he joined the Faculty of the Department of Electrical Engineering, Jadavpur University, as a Lecturer. He then joined the Department of Electrical Engineering, Bengal Engineering College (Deemed University), Howrah, India, in 1990 as an Assistant Professor. His fields of interest are electrical machines and drives, power electronics, and instrumentation. He has authored more than 30 technical papers, which have beeen published in India and abroad.

Mr. Basak was awarded the University Gold Medal and the B. C. Roy Gold Medal for his academic performance. He received the Gold Medal of the Institution of Engineers (India) for the best paper of 1986. He is a member of the Institution of Engineers (India), IEEE Industry Applications Society, and Institution of Standard Engineers (India) and a life member of the Solar Energy Society of India and the Indian Society of Technical Education.

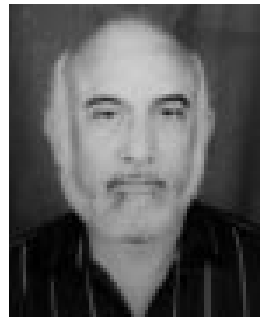

Debi Prasad Sen Gupta received the B.Sc. degree (with honors) in physics from Presidency College, Calcutta, India, and later studied electrical engineering at the Indian Institute of Technology, Kharagpur, India. He received the Ph.D. degree from the University of Liverpool, Liverpool, U.K.

He taught for five years at the University of Liverpool, before joining the Indian Institute of Science, Bangalore, India, where he taught and carried out research as a Professor until his retirement in 1996.

Currently, he is continuing in an Emeritus position at the Indian Institute of Science. His main areas of research are power system stability and control and Indian energy problems, particularly in the power sector. He has had research links with a number of British universities and, at present, with the University of Illinois, Urbana-Champaign. He has published several research papers and books.

Dr. Sen Gupta is the recipient of awards for teaching excellence from Liverpool University and the Indian Institute of Science. 